\title{
Orientaciones para la elaboración y actualización de Recomendaciones Clínicas de la Sociedad de Anestesiología de Chile
}

\author{
Guidelines for the development and update of Clinical \\ Recommendations of the Anesthesiology Society of Chile
}

Waldo Merino Urrutia', Katia Guerra Holstein², Milca Villagrán Schmidt³

\section{Introducción}

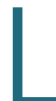

as guías de práctica clínica resumen y evalúan la evidencia disponible sobre un tema en particular, - con el objetivo de ayudar a los profesionales de la salud en la selección de las mejores estrategias para enfrentar una situación clínica específica, en un paciente individual, considerando los potenciales riesgos y beneficios de sus decisiones.

La calidad y rigurosidad de estas publicaciones han presentado en el tiempo importantes progresos y mayores exigencias metodológicas a propósito del impacto que se espera de ellas[1]. "Deben promover intervenciones con claro beneficio para el paciente y desalentar aquellas que no redundan en reducir la morbilidad, mortalidad y mejoran el resultado final. Además, deben constituir la mejor herramienta para homogeneizar la práctica clínica, es decir, disminuir la variabilidad clínica indeseada y actualizarla con el más reciente estado del arte en un tópico específico"[2]. Por ello, es una política deseada el que se actualicen periódicamente. Estas razones, entre otras, son las que han invitado a distintas instituciones a apoyar el desarrollo de este tipo de publicaciones como son las sociedades científicas, oficinas gubernamentales, centros clínicos y universidades. También representan la posición oficial de las sociedades científicas y agencias gubernamentales sobre determinado tema, respaldados por la mejor evidencia presentada por expertos y validada por estas instituciones[1],[2].

Aunque no reemplazan el juicio clínico del anestesiólogo, que conserva el derecho de autonomía, expresado en la responsabilidad intransferible de administrar anestesia y proveer los cuidados perioperatorios necesarios a sus pacientes, las Guías de Práctica Clínica han impactado profundamente en la práctica profesional y en la medicina de sus respectivos países, han colaborado en el desarrollo de políticas institucionales y han facilitado la toma de decisiones de manera transparente para los pacientes y sus familias.

Así, la Sociedad de Anestesiología de Chile (SACH) no ha estado ajena a este proceso y se ha planteado como una política de la institución la elaboración de Recomendaciones Clínicas (RCs) entendiendo que "la medicina basada en la evidencia surge por la necesidad de sustentar la práctica clínica en algo de mayor

Departamento de Cirugía, Traumatología y Anestesiología. Universidad de La Frontera. Temuco, Chile.

2 Hospital Roberto del Río, Anestesiólogo Hospital Clínico Pontificia Universidad Católica de Chile. Docente Pontificia Universidad Católica de Chile. Santiago, Chile.

3 Hospital Hernán Henríquez Aravena. Temuco, Chile.

Comité de Recomendaciones Clínicas de la Sociedad de Anestesiología de Chile.

\section{ORCID}

https://orcid.org/0000-0002-4956-8444

Fecha de recepción: 12 de diciembre de 2019

Fecha de aceptación: 15 de diciembre de 2019

Correspondencia:

Dr. Waldo Merino

Email: wamerino@gmail.com 
fortaleza que la experiencia, y de ella se derivan las recomendaciones clínicas"[2]. Estas RCs tienen como propósito respaldar el trabajo profesional de nuestros asociados a lo largo del país, con estándares de calidad adecuados a los tiempos, señalando la mejor evidencia disponible, para un mejor desempeño clínico y profesional, pensando en el beneficio de nuestros pacientes y de la salud pública de nuestro país. De esta manera la SACH da cumplimiento a su estatuto que establece en su artículo segundo, como un objetivo fundamental el "perfeccionar y ampliar los conocimientos de la especialidad, estudiar y divulgar problemas científicos $y$, en general, propender al progreso de la Anestesia".

A la fecha contamos con 13 RCs de la SACH y la participación en las Guías de Práctica Clínica del MINSAL:

1. Mayo de 2016: Recomendaciones clínicas cuidado perioperatorio anestésico y manejo del dolor en la cirugía bariátrica.

2. Junio de 2011: Recomendaciones clínicas de reanimación cardiopulmonar perianestésica del adulto.

3. Junio de 2011: Recomendaciones clínicas para el manejo de la anafilaxia en anestesia.

4. Junio de 2011: Recomendaciones clínicas para la anestesia fuera de pabellón.

5. Junio de 2010: Recomendaciones clínicas para el manejo de la crisis de hipertermia maligna y para el manejo del paciente susceptible de hipertermia maligna.

6. Junio de 2010: Recomendaciones clínicas para el manejo de estupefacientes y sustancias controladas.

7. Diciembre de 2007: Recomendaciones clínicas para el manejo del dolor agudo postoperatorio en Adultos.

8. Octubre de 2007: Recomendaciones clínicas para la participación de sus asociados en actividades clínicas de manejo del dolor en sus diversas formas de manifestación.

9. Octubre de 2007: Recomendaciones clínicas para el manejo del dolor crónico oncológico.

10. Octubre de 2007: Recomendaciones clínicas para el manejo del dolor crónico no oncológico.

11. Octubre de 2007: Recomendaciones clínicas para el manejo del dolor agudo en niños.

12. Octubre de 2007: Analgesia del parto serie Guías Clínicas MINSAL № 54, 2007.

13. Marzo de 2007: Recomendaciones clínicas de evaluación preanestésica.

14. Agosto de 2007: Recomendaciones de disponibilidad y uso de monitorización intraoperatoria.
A esta lista, durante los años 2018 y 2019, se han incorporado las siguientes actualizaciones[3]-[5]:

- Recomendación Clínica "Manejo del Dolor Agudo Perioperatorio en Niños".

- Recomendación Clínica: Disponibilidad y Uso de Monitorización Perioperatoria.

- Recomendación Clínica: Evaluación preoperatoria.

En 2017 la SACH acordó que las nuevas Rcs se formularán de acuerdo a una metodología, formato en común, que señale objetivos generales, ámbito de influencia, sustento técnico, entre otros. En el marco de claros criterios técnicos y exigencias metodológicas actualizadas y fundamentados en el nivel de evidencia de la literatura.

Además, a partir de este año la SACH ha decidido conformar un comité permanente de RCs que sostenga una política de desarrollo, formulación y renovación de ellas, dando cuenta de nueva evidencia, avances tecnológicos e interés de la propia Sociedad de Anestesiología, sus socios y pacientes.

El comité tendrá como función específica determinar las nuevas RCs a propósito de la razón que invita a su formulación y la influencia que se espera de ellas. Además, determinar la metodología y diseño de las Gcs, facilitará la conformación de grupos de trabajo o solicitará a determinados centros la formulación y/o renovación de las Rcs para dar respuesta a esta política de la SACH. Para el comité, las RCs no solo pretenden apoyar la toma de decisiones de los anestesiólogos del país, en cuanto a los cuidados perioperatorios de sus pacientes, sino que también señalar las condiciones de infraestructura y equipamiento que den garantías para un buen desempeño profesional y definir aquellas conductas que resultan apropiados para circunstancias clínicas específicas, considerando la mejor evidencia científica disponible, ofreciendo a las autoridades sanitarias, administradores de salud pública y privada, pacientes y familiares, información relevante para la toma de decisiones.

Es importante señalar que las RCs son una exhaustiva revisión de expertos, donde se puede reconocer algunas condiciones de equipamiento e infraestructura que son necesarias para cumplir un desempeño profesional acorde a lex artis. Estas condiciones, en general, son una responsabilidad exclusiva y excluyente del administrador o gestor de los recursos en el sector público y privado. Por esta razón la SACH recomienda a sus asociados hacer presentaciones escritas para que los administradores y gestores se mantengan razonablemente informados en estas materias. Las comunicaciones respectivas se pueden canalizar a 
través de la SACH, ello permite conducir y facilitar los procesos en las instituciones sanitarias que sostengan condiciones de trabajo y clínicas deficitarias.

\section{En cuanto a la metodología...}

El Ministerio de Salud (MINSAL) ha desarrollado una definición precisa de los contenidos y condiciones de calidad que deben cumplir las Guías de Práctica Clínica y Recomendaciones Clínicas (RCs) en nuestro país, ha generado diversos documentos para guiar a los grupos técnicos en el proceso de formulación sintetizados en el "Manual Metodológico. Desarrollo de Guías de Práctica Clínica"[6].

El MINSAL ha desarrollado esta política en el contexto del Régimen de Garantías Explícitas (AUGEGES), y ha elaborado un manual metodológico incorporando la metodología GRADE. Este sistema de graduación de recomendaciones es explícita, fácil de entender, transparente y pragmática, y fue llamado "The Grading of Recommendations Assessment, Development and Evaluation" (GRADE), el cual ha sido adoptado por más de 80 organizaciones elaboradoras de guías clínicas como: World Health Organization, Cochrane Collaboration, National Institute for Clinical Excellence (NICE), The Scottish Intercollegiate Guidelines Network (SIGN), Canadian Task Force on Preventive Health Care, British Medical Journal (BMJ), The Canadian Agency for Drugs and Technologies in Health (CADTH), UpToDate, entre otras[6].

"Las ventajas del sistema GRADE sobre otros sistemas de graduación de recomendaciones radican en que este fue desarrollado por un grupo amplio y representativo de elaboradores de guías a nivel internacional, posee una clara separación entre la calidad de la evidencia y la fuerza de la recomendación, posee un evaluación explícita de la importancia de los desenlaces para estrategias alternativas, posee un sistema de evaluación de la calidad de la evidencia claro y explícito, realiza las recomendaciones a través de un proceso transparente, toma de forma explícita los valores y preferencias de los pacientes, la interpretación de la fuerza de la recomendación para los clínicos, pacientes y tomadores de decisión es clara y pragmática y también puede ser utilizada para la elaboración de evaluación de tecnologías sanitarias y revisiones sistemáticas"[6]-[8].

Así, para la evaluación de la evidencia y formulación de las RCs de la SACH se utilizó el sistema GRADE recomendado por el MINSAL.

A través de estas RCs la SACH contribuye en el cumplimiento de su propósito, de su responsabilidad ética y su compromiso con el país, desarrollando y participando en actividades que aporten al mejoramiento de la comunidad y la salud pública, difundiendo y promoviendo los conocimientos científicos, poniendo a disposición de los pacientes, autoridades sanitarias y anestesiólogos, información pertinente para apoyar la práctica de los colegas, en beneficio de sus pacientes.

Así, este documento tiene como propósito entregar orientaciones para la elaboración de las Recomendaciones Clínicas de la SACH.

\section{Objetivo general}

Entregar una guía para la elaboración de recomendaciones clínicas que, con rigurosidad metodológica y alto estándar de calidad, señalen las condiciones de infraestructura y equipamiento necesarias para entregar garantías que permitan un buen desempeño profesional, y que ofrezcan orientaciones con respecto a las mejores estrategias que deberían ayudar a tomar decisiones en el ejercicio clínico de los anestesiólogos del país, como también ofrecer información relevante para autoridades sanitarias, administradores públicos y privados, pacientes y familiares.

\section{Objetivos específicos}

- Estandarizar los elementos conceptuales y metodológicos más relevantes sobre la formulación de la recomendación clínica, que considere:

- Las mejores evidencias científicas disponibles en la formulación de las recomendaciones.

- Métodos de consenso participativos, transparentes y explícitos, en la síntesis de la evidencia como en la declaración, elaboración y redacción de las recomendaciones.

- Establecer un formato reconocible para los usuarios.

- Estandarizar la conformación y funciones de los equipos de trabajo responsables de la elaboración de la recomendación.

- Definir el formato de trabajo y características de los revisores de las RCs.

\section{Elaboración de las Recomendaciones}

1. Conformación y funciones de los equipos de trabajo responsables de la elaboración de la recomendación.

- El comité de RCs invitará a participar a sus socios 
especialmente o a profesionales expertos en la conformación de grupos de trabajo para la elaboración de una RCs en particular. Estos grupos también se pueden conformar por decisión e integrantes de otros comités.

- De acuerdo al tópico de interés solicitará a determinados centros o socios en particular la resposabiliad de la formulación y/o renovación de las Rcs.

- En lo posible se sugiere conformar grupos por afinidad (centros clínicos, comités de la sociedad, afinidad por temas de interés) de número impar para la resolución de controversias.

- Recomendamos que uno de los integrantes del grupo coordine el trabajo de los integrantes, en cuanto a tareas, cumplimiento de plazos, establecer cronograma de reuniones de trabajo, entre otros.

- Se establecerá un miembro del comité de RCs como nexo con el grupo de trabajo, para asesoría y colaboración en la elaboración del documento.

- Para nuevas RCs el tópico es definido por el directorio de la SACH, el comité de RCs o de la decisión de elaborar una RC de un grupo de socios o comité de la SACH, propio de su interés.

2. Definición del objeto de la evaluación.

Esto implica haber identificado con claridad:

- Los tipos de pacientes de interés.

- Las intervenciones o factores a evaluar.

- Los desenlaces de interés o medidas de resultado a los que se refiere la evaluación.

\section{Búsqueda amplia de estudios científicos}

Una vez definido el objeto de la RC es necesario efectuar una búsqueda bibliográfica o de la evidencia disponible de manera ordenada. Deberá explicitarse en el documento con la determinación de palabras clave en consenso con todos los integrantes del grupo de trabajo. Recomendamos desarrollar la búsqueda considerando los siguientes aspectos.

\section{a. Definir el problema clínico}

Una vez planteada la pregunta de investigación, debemos transformarla en un tema o pregunta de búsqueda. El éxito de la búsqueda dependerá de la pregunta de búsqueda. Se sugiere especificar o focalizar lo más posible, sugerimos facilitar este trabajo a partir de la técnica y acrónimo PICoR. b. Identificar tipo de trabajo de investigación puede responder su pregunta

- Estudios de terapia, prevención, daño, etiología: revisiones sistemáticas, ensayos clínicos randomizados, estudios de cohorte, estudios de casos y controles.

- Estudios de diagnóstico: revisiones sistemáticas, estudios de test diagnósticos.

- Estudios de pronóstico: revisiones sistemáticas, estudios de cohorte, series de casos.

- Estudios de impacto económico: estudios de costo-efectividad o costo-utilidad, evaluaciones de tecnologías sanitarias.

c. Identificar fuentes de búsqueda

La búsqueda puede considerar las siguientes fuentes:

- Generales

ACP Journal Club (www.acpjc.org)

Scientific American Medicine (www.samed.com)

Medscape (www.medscape.com)

National Guideline Clearinghouse (www.guideline.gov)

- Secundarias (sitios de información filtrada)

American College of Physicians (www.acponline. org)

UpToDate (www.uptodate.com)

Clinical Evidence (www.clinicalevidence.com)

- Bases de datos:

Embase (www.embase.com/login)

Medline (www.pubmed.com)

Cochrane Library (http://www.cochranelibrary. com o www.bvs.cl)

Lilacs (www.bvs.cl)

Scielo (https://scielo.conicyt.cl o www.bvs.cl)

- Revistas específicas.

\section{d. Diseñar estrategias de búsqueda}

Mecanismos de búsqueda que permitan una revisión exhaustiva y sistemática de la literatura científica, para lo cual se revisarán diferentes fuentes de información.

\section{e. Registrar resultados de búsqueda}

La búsqueda ordenada deberá explicitarse en el documento con la determinación de palabras clave en consenso con todos los integrantes del grupo de trabajo.

$\begin{array}{ll}\text { Paciente/población } & \text { ¿De qué grupo requiero información? } \\ \text { Exposición/intervención } & \text { ¿Cuál es el evento médico cuyo efecto quiero estudiar? } \\ \text { Comparación } & \text { ¿Quiero compararlo? } \\ \text { Resultados } & \text { ¿Cuál es el efecto de la intervención? }\end{array}$


Ejemplo:

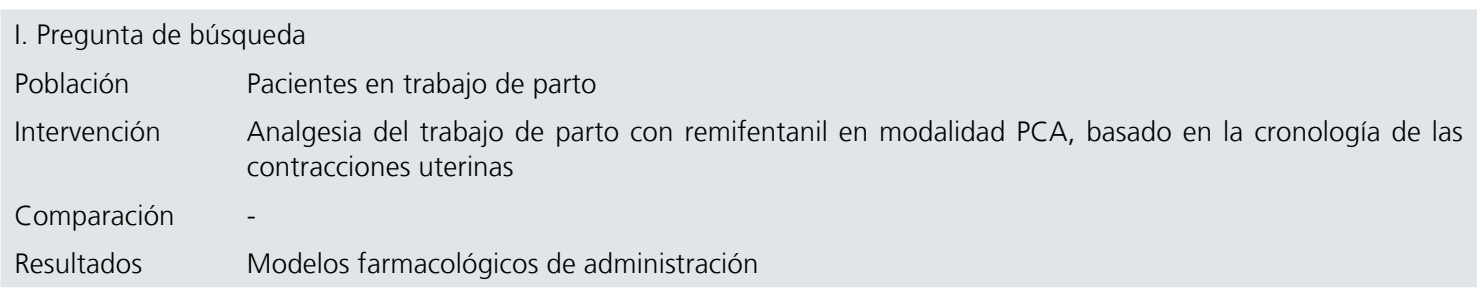

¿Existen sistemas de administración de remifentanil en modalidad PCA (analgesia controlada por el paciente) para la analgesia del trabajo de parto basado en la cronología de las contracciones uterinas?

\section{Tipo de trabajo de investigación}

Estudios de terapia (ensayos clínicos y/o revisiones sistemáticas-metaanálisis).

III. Fuente de búsqueda PubMed

IV. Estrategia de búsqueda recurrirá al análisis de estudios primarios y revisiones narrativas. Lo anterior no obsta para que el análisis de las revisiones sistemáticas disponibles incluya, en caso necesario, el examen de los estudios primarios contenidos en ellas[7].

5. Criterios explícitos de elegibilidad de los estudios[6], relacionados con:

- Correspondencia con el objeto de evaluación definido: Ante todo, se seleccionará estudios que proporcionen evidencias directas, esto es, investigaciones realizadas en poblaciones o muestras equivalentes o asimilables a la población de inte-

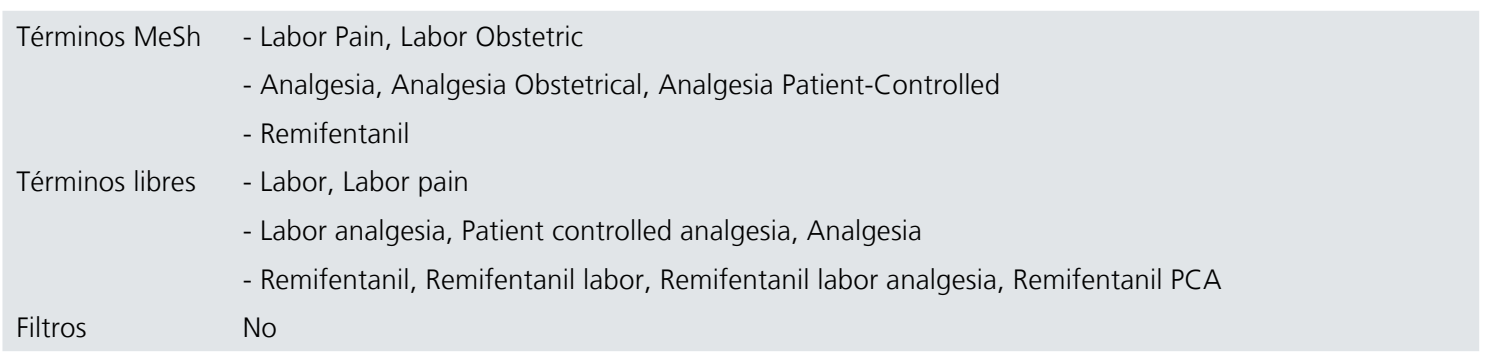

V. Registro de la búsqueda. Ejemplo: Pub Med Central.

- Número total de resultados.

Frase de búsqueda $\rightarrow 178$

- Número de potenciales artículos de interés.

Cribado por títulos $\rightarrow 65$

- Número de artículo que le servirán para justificar su pregunta.

\section{Cribado por Abstract $\rightarrow 29$}

4. La búsqueda privilegiará la identificación de revisiones sistemáticas disponibles en la literatura

Este criterio tiene por objetivo limitar la introducción de sesgos en el análisis. En caso que tales revisiones no existan o sean de calidad insuficiente, se rés, en las que el factor o intervención evaluado sea el mismo, y que hayan medido las mismas variables de resultado.

- Diseño de investigación: Los diseños elegibles deben ser aquellos que provean la evidencia de mayor validez científica disponible sobre la materia evaluada. Análisis de validez interna: análisis del riesgo potencial de sesgos, confusión y azar en sus resultados, de acuerdo a criterios preestablecidos, y aplicado por igual a todos los estudios seleccionados.

6. Proceso de síntesis de las evidencias obtenidas de los estudios[8]. Esto incluye:

Grados de evidencia y niveles de recomendación utilizados en la RCs. Así, para la evaluación y redac- 


\begin{tabular}{|c|c|c|c|}
\hline Search & Add to builder & Query & Items found \\
\hline Search & Add to builder & Query & Items found \\
\hline$\# 29$ & Add & $\begin{array}{l}\text { Search ((((((labor) OR labor pain) OR "Labor Pain"[Mesh]) OR "Labor, Obstetric"[Mesh])) AND } \\
\text { ((((((labor analgesia) OR patient controlled analgesia) OR "Analgesia"[Mesh]) OR analgesia) OR } \\
\text { "Analgesia, Obstetrical"[Mesh]) OR "Analgesia, Patient-Controlled"[Mesh])) AND (((((remifentanil) OR } \\
\text { remifentanil labor) OR remifentanil labor analgesia) OR remifentanil pca) OR "remifentanil" } \\
\text { [Supplementary Concept])) }\end{array}$ & $\underline{178}$ \\
\hline$\underline{\# 28}$ & $\underline{\text { Add }}$ & $\begin{array}{l}\text { Search ((((((labor) OR labor pain) OR "Labor Pain"[Mesh]) OR "Labor, Obstetric"[Mesh])) AND } \\
\text { ((((((labor analgesia) OR patient controlled analgesia) OR "Analgesia"[Mesh]) OR analgesia) OR } \\
\text { "Analgesia, Obstetrical"[Mesh]) OR "Analgesia, Patient-Controlled"[Mesh])) AND (((((remifentanil) OR } \\
\text { remifentanil labor) OR remifentanil labor analgesia) OR remifentanil pca) OR "remifentanil" } \\
\text { [Supplementary Concept]) }\end{array}$ & $\underline{178}$ \\
\hline$\# 27$ & $\underline{\text { Add }}$ & $\begin{array}{l}\text { Search ((((remifentanil) OR remifentanil labor) OR remifentanil labor analgesia) OR remifentanil pca) } \\
\text { OR "remifentanil" [Supplementary Concept] }\end{array}$ & $\underline{4371}$ \\
\hline \#26 & Add & $\begin{array}{l}\text { Search ((((labor analgesia) OR patient controlled analgesia) OR "Analgesia"[Mesh]) OR analgesia) OR } \\
\text { "Analgesia, Obstetrical"[Mesh]) OR "Analgesia, Patient-Controlled"[Mesh] }\end{array}$ & $\underline{74920}$ \\
\hline \#25 & Add & Search (((labor) OR labor pain) OR "Labor Pain"[Mesh]) OR "Labor, Obstetric"[Mesh] & 968395 \\
\hline$\# 24$ & Add & Search "remifentanil" [Supplementary Concept] & $\underline{2842}$ \\
\hline \#22 & $\underline{\text { Add }}$ & Search remifentanil pca & $\underline{119}$ \\
\hline$\# 21$ & Add & Search remifentanil labor analgesia & $\underline{178}$ \\
\hline \#20 & Add & Search remifentanil labor & $\underline{215}$ \\
\hline$\# 19$ & $\underline{\text { Add }}$ & Search remifentanil & $\underline{4371}$ \\
\hline$\# 18$ & $\underline{\text { Add }}$ & Search "Analgesia, Patient-Controlled"[Mesh] & $\underline{4068}$ \\
\hline$\# 16$ & Add & Search "Analgesia, Obstetrical"[Mesh] & $\underline{3599}$ \\
\hline$\# 14$ & $\underline{\text { Add }}$ & Search analgesia & $\underline{74920}$ \\
\hline$\# 13$ & Add & Search "Analgesia"[Mesh] & $\underline{39694}$ \\
\hline$\# 11$ & $\underline{\text { Add }}$ & Search patient controlled analgesia & 9388 \\
\hline$\# 10$ & Add & Search labor analgesia & $\underline{7742}$ \\
\hline$\underline{\# 9}$ & $\underline{\text { Add }}$ & Search "Labor, Obstetric"[Mesh] & $\underline{43656}$ \\
\hline$\# 8$ & $\underline{\text { Add }}$ & Search "Labor Pain"[Mesh] & $\underline{914}$ \\
\hline$\# 7$ & Add & Search labor pain & $\underline{5720}$ \\
\hline \#6 & Add & Search labor & 968395 \\
\hline
\end{tabular}

ción de las recomendaciones se utilizó el sistema GRADE recomendado por el MINSAL. Este clasifica la calidad de la evidencia como sigue:

Nivel de la calidad de la evidencia

Alto: Estamos relativamente seguros de que el efecto real de la intervención se encuentra cerca de nuestra estimación.

Moderado: El efecto real de la intervención probablemente se encuentra cerca de nuestra estimación, pero existe la posibilidad de que sea sustancialmente diferente.

Bajo: El efecto real de la intervención puede ser sustancialmente diferente de nuestra estimación.

Muy bajo: Es probable que el efecto real de la intervención sea sustancialmente diferente de nuestra estimación.

El curso de acción de acuerdo a la fuerza de la recomendación según el sistema GRADE[8].
A. Recomendaciones fuertes:

La alternativa recomendada puede ser seguida con todos o casi todos los pacientes. Una conversación detallada con el paciente o una revisión cuidadosa de la evidencia que fundamenta la recomendación pudiera no ser necesaria. Se cumplen TODAS estas condiciones:

- Calidad de la evidencia: Alta o moderada (o baja o muy baja en circunstancias excepcionales).

- Balance de beneficios y riesgos: Una alternativa es claramente superior.

- Valores y preferencias de los pacientes: Todos o casi todos los pacientes informados toman la misma decisión.

- Consideraciones de recursos: El costo de la intervención está plenamente justificado.

B. Recomendaciones débiles:

Si bien la alternativa recomendada es apropiada para la mayoría de los pacientes, la decisión debiera 
ser individualizada, idealmente mediante un enfoque de decisiones compartidas. Se cumple ALGUNA de estas condiciones:

- Calidad de la evidencia: Baja o muy baja.

- Balance de beneficios y riesgos: El balance de beneficios y riesgos es cercano.

- Valores y preferencias de los pacientes: Existe variabilidad o incertidumbre respecto de lo que decidirán pacientes informados.

- Consideraciones de recursos: El costo de la intervención pudiera no estar justificado en algunas circunstancias.

\section{Redacción de las recomendaciones}

Como hemos mencionado, la formulación de las RCs se basa en el manual metodológico del MINSAL, que incorpora la metodología GRADE[6]. Se inicia con la conformación de un grupo de trabajo, el cual adquiere un compromiso con la SACH de desarrollar la RC. Este grupo de trabajo inicia una búsqueda sistemática en la literatura médica, identificando guías de práctica clínica, revisiones sistemáticas y estudios primarios, aplicando filtros metodológicos de acuerdo con lo descrito previamente. Luego, se procede a la selección y síntesis de la evidencia, y se evalúa la calidad de la evidencia, con el objetivo de dar forma a la redacción de la recomendación. cada recomendación debe ser escrita de manera clara y precisa, seguida del nivel de la calidad de la evidencia y el tipo de recomendación según metodología GRADE. A continuación, algunos ejemplos:

Sugerimos en lo posible redactar la sentencia en términos positivos. Los siguientes cinco ejemplos son extraídos de la RC "Manejo del Dolor Agudo Perioperatorio en Niños"[6].

\section{Ejemplo 1:}

Recomendamos utilizar escalas validadas en niños para la evaluación del dolor postoperatorio, de acuerdo con las características de cada paciente.

\section{Calidad baja/Recomendación fuerte}

Sugerimos citar la bibliografía en toda circunstancia sea la evidencia fuerte o muy débil. Sugerimos citar los estudios originales cuando la afirmación es controvertida o se basa en un solo estudio original o en revisiones de calidad insuficiente.

\section{Ejemplo 2:}

Recomendamos el uso de AINES por períodos cortos de tiempo y en mayores de 6 meses. Recomendamos no usar aspirina en niños $(4,24,28)$.

\section{Calidad alta/Recomendación fuerte}

Ejemplo 3:

Recomendamos usar dexametasona endovenosa como adyuvante analgésico en el perioperatorio de niños $(13,24,28)$.

\section{Calidad moderada/Recomendación fuerte}

Ejemplo 4:

Recomendamos utilizar metamizol como analgésico, como primera línea o alternativa (43).

\section{Calidad baja/Recomendación débil}

A continuación de la recomendación, cuando parece necesario, para sugerir una dosis o a propósito de una intervención controvertida, novedosa o compleja, se puede redactar un comentario con el fin de explicar con mayor detalle el sustento de la recomendación.

\section{Ejemplo 5:}

Recomendamos usar metadona endovenosa sólo en bolo, para el manejo perioperatorio del dolor moderado a severo en niños mayores de 1 año (13).

\section{Calidad de la evidencia baja/Recomendación fuerte}

Comentario: Metadona presenta, además de su efecto opioide de larga duración, un efecto de antagonista NMDA, lo que puede hacerla atractiva en el contexto perioperatorio. Su uso se recomienda en bolo endovenoso y en niños mayores de 1 año. Presenta como desventaja una inadecuada e irregular concentración plasmática, debido a una rápida redistribución tras un bolo endovenoso (13).

Dosis metadona: 50-100 ug/kg endovenoso en bolo, cada 6 h.

No siempre es necesario establecer la calidad y la fuerza de la recomendación si esta descansa en la opinión de los expertos.

\section{Resolución de controversias:}

Las definiciones con respectos a los puntos anteriores, sin duda, pueden llevar a diferencias entre los autores[7]. Estas controversias pueden encontrarse en la apreciación de:

- La validez interna de los estudios.

- La validez externa de los estudios para el contexto chileno.

- La relevancia clínica de los resultados.

- El balance entre los desenlaces favorables y desfa- 
vorables.

- El rol que cabe a los pacientes.

- Las condiciones de aplicabilidad o el impacto esperado de las recomendaciones.

- Las evidencias encontradas resulten contradictorias o las condiciones locales no hagan aplicable la recomendación.

Para resolver estas diferencias se deben aplicar métodos de consenso, que deben asegurar:

- Representatividad: deben incluir todos los autores y eventualmente, algún miembro del comité de RCs.

- Que todos los miembros del grupo a cargo de la elaboración del documento dispongan del mismo nivel de información básica para la toma de decisiones.

9. Además es recomendable:

- La pertinencia de generalizar los resultados obtenidos de la literatura, a nuestra población de interés.

- Establecer la fecha de validez del documento.

- Declaración de conflictos de interés de los miembros del grupo a cargo de la elaboración del documento, cuando corresponda.

10. Proceso de revisión:

- Los procesos de revisión de las propuestas de RCS se realizarán por el comité al momento de la primera entrega del texto para revisión. Cada recomendación tendrá un plazo para efectuar las modificaciones necesarias de ocho semanas para nueva entrega.

- Una vez superado el proceso de revisión, las Rcs serán publicadas en la Revista Chilena de Anestesia.

\section{Formato sugerido para las recomendaciones clínicas de la SACH}

(Este formato es modificable de acuerdo a las características propias de cada recomendación)

1. Sección Inicial:

1.1. Tapa con título en forma destacada, identificación y logo de la SACH.

1.2. Identificación del grupo de trabajo a cargo de la formulación de la GPC, y su coordinador responsable. Identificar cargo y vinculación institucional.

1.3. Fecha de presentación de la RC.

1.4. Índice de contenidos (incluyendo número de página).

2. Resumen estructurado:
2.1. Propósito de la GPC.

2.2. Metodología.

2.3. Síntesis de recomendaciones.

3. Lista de abreviaturas.

4. Definición de términos.

5. Declaración de conflicto de interés de los participantes.

6. Desarrollo de la RC.

6.1. Introducción.

6.2. Objetivos.

6.2.1. Escenarios clínicos a los que se refiere la RC y condiciones de aplicación.

6.2.2. Eventuales situaciones en las que no es aplicable la RC.

6.3. Métodos:

6.3.1. Identificación de documentos fuente. Síntesis de evidencia:

6.3.1.1. Identificación de prestaciones vinculadas al problema de salud.

6.3.1.2. Efectividad de las intervenciones.

6.3.1.3. Efectos adversos de las intervenciones.

6.3.1.4. Condiciones de calidad de las intervenciones.

6.3.2. Métodos de consenso utilizados para la formulación de recomendaciones.

6.3.3. Grados de evidencia y niveles de recomendación utilizados en la RCs.

6.3.4. Consideraciones ético-sociales.

7. Recomendaciones:

7.1. Las recomendaciones clave, de mayor importancia, serán destacadas en el texto y claramente identificables.

7.2. Cada recomendación contendrá, al final de la misma:

7.2.1. Una identificación del documento fuente en el que se sustenta (no aplicable a recomendaciones basadas exclusivamente en la opinión de expertos).

7.2.2. Clasificación del nivel de la evidencia.

8. Flujogramas/algoritmos de decisión.

9. Herramientas de ayuda para la decisión: versiones abreviadas de bolsillo, posters, material educativo, folletos para pacientes (si se han definido, se deben identificar y anexar al documento).

10. Repercusiones económicas potencialmente relevantes de la aplicación de la RC.

11. Referencias (toda la bibliografía debe encontrarse vinculada con el texto de la GPC, con un número asociado de acuerdo al formato de la Revista Chilena de Anestesia).

12. El formato en lo que se refiere al tipo y tamaño de letra, gráficos, figuras e imágenes se deben incorporar de acuerdo a lo señalado en la guía de autores de Revista Chilena de Anestesia. 


\section{Referencias}

1. Recommendations for guidelines productions. A document for task forces members responsible for the production un updating of ESC Guidelines. Committe for Practice Guidelines of the European Society of Cardiology. https://www.escardio.org/ static_file/Escardio/Guidelines/ about/ESC_Guidelines_for_Guidelines_Update_2012_for_web. pdf

2. Bustamante R. Recomendaciones Clínicas [Editorial]. Rev Chil Anest. 2012;41:163-5.

3. Campos T, Eulufi S, Fajardo Razmilic MA, Guerra Hollstein KE, Pérez Díaz IMM. Recomendación Clínica "Manejo del Dolor Agudo Perioperatorio en Niños". Rev Chil Anest.
2018;47(1):46-63. https://doi. org/10.25237/revchilanestv47n01.09.

4. Egaña Tomic J, Fuenzalida Soler $\mathrm{P}$, Jiménez Esperidión $\mathrm{C}$, Jara Schnettler Á, Maldonado Caniulao F, Penna Silva A, et al. Recomendación Clínica: Disponibilidad y Uso de Monitorización Perioperatoria. Revista Chilena de Anestesia. 2018;47(2):13744. http://dx.doi.org/10.25237/ revchilanestv47n02.12

5. Fuentes HR, Nazar JC, Vega GP, Stuardo MC, Parra PA, Merino UW. Recomendación clínica: evaluación preoperatoria. Rev Chil Anest 2019; 48: 182-193 http:// dx.doi.org/10.25237/revchilanestv48n02.17

6. Ministerio de Salud. Subsecertaria de Salud. División de control y prevención de enfermedades.
Departamento Secretaria AUGE y de coordinación evidencial y metodológica. Manual Metodológico. Desarrollo de Guías de Práctica Clínica. Santiago, Chile; 2014.

8. Higgins JP, Green S, editors. Cochrane Handbook for Systematic Reviews of Interventions Version 5.1.0 [updated March 2011]. The Cochrane Collaboration, 2011. www.cochranehandbook.org

9. Neumann I, Pantoja T, Peñaloza B, Cifuentes L, Rada G. El sistema GRADE: un cambio en la forma de evaluar la calidad de la evidencia y la fuerza de recomendaciones. Rev Med Chile. 2014 May;142(5):630-5. https://doi.org/10.4067/ S0034-98872014000500012 PMID:25427021 